\title{
Medium-Chain Triglycerides in Infant Formulas and their Relation to Plasma Ketone Body Concentrations
}

\author{
PAUL Y. K. WU, JOHN EDMOND, NANCY AUESTAD, SAVITRI RAMBATHLA, JOHN BENSON, \\ AND TOM PICONE
}

The Neonatology Division, Department of Pediatrics, University of Southern California School of Medicine, LAC-USC Medical Center, Department of Biological Chemistry, UCLA School of Medicine, Los Angeles, California and Nutritional Division, Ross Laboratories, Columbus, Ohio

\begin{abstract}
A mild ketosis is known to prevail in the mother, fetus, and newborn infant during the 3rd trimester and in the early neonatal period. It has been shown that during an equivalent period in the rat ketone bodies are readily oxidized and serve as key substrates for lipogenesis in brain. Since medium-chain triglycerides are known to be ketogenic, preterm infants may benefit from dietary medium-chain triglycerides beyond the point of enhanced fat absorption. Our objective was to determine the ketogenic response in preterm infants (gestational age: $33 \pm 0.8$ wk) fed three different isocaloric formulas by measuring the concentrations of 3-hydroxybutyrate and acetoacetate in the plasma of these infants. At the time of entrance to the study the infants were receiving $110 \mathrm{kcal} / \mathrm{kg} / 24 \mathrm{~h}$. Study I (11 infants): the infants were fed sequentially in the order; PM 60/40 (PM), Special Care Formula (SCF), and Similac 20 (SIM). In SCF greater than $50 \%$ of the fat consists of medium-chain length fatty acids while PM and SIM contain about $25 \%$. The concentration of 3-hydroxybutyrate in plasma was significantly higher when infants were fed SCF than PM and SIM $[0.14 \pm 0.03,0.06 \pm 0.01$, and $0.05 \pm 0.01 \mathrm{mM}$, respectively $(p<0.01)$ ]. Study II $(12$ infants); the infants were fed SCF, then SIM, or the reverse. The concentration of acetoacetate in plasma was $0.05 \pm 0.01$ and $0.03 \pm 0.01 \mathrm{mM}$ when infants were fed SCF and SIM, respectively $(0.1>p>0.05)$. The concentrations of 3-hydroxybutyrate in plasma were similar to those measured in study I for the respective formulas. The increased plasma levels of 3-hydroxybutyrate and total ketone bodies when SCF was fed indicate that SCF promotes a mild ketosis in preterm infants similar to that reported in breast-fed term infants. (Pediatr Res 20: 338341, 1986)
\end{abstract}

Abbreviations

AcAc, acetoacetate

3HB, 3-hydroxybutyrate

MCT, medium-chain triglycerides

PM, PM 60/40

SCF, Similac Special Care Formula

SIM, Similac 20

Received March 29, 1985; accepted December 12, 1985

Address for correspondence and reprints Paul Y. K. Wu, M.D., Women's Hospital, L-919, 1240 N. Mission Road, Los Angeles, CA 90033.

Supported in part by a grant from The Nutritional Division, Ross Laboratories, Columbus, $\mathrm{OH}$
The past decade has witnessed a major change in our concept of ketone bodies from a limited role as alternative oxidative substrates in muscle and brain to a role as key substrates for tissue respiration and lipid synthesis. Recent studies with animal models (1-4) and in human infants (5-9) suggest that the ketone bodies, AcAc and $3 \mathrm{HB}$, may be particularly important in the developing infant. Several investigators have demonstrated that AcAc and $3 \mathrm{HB}$, in addition to serving as general oxidative substrates, are preferentially incorporated into sterols and fatty acids in the neonatal rat brain $(1,2,10)$. This occurs during the "brain growth spurt" when cell proliferation and myelination are major developmental events. In the human myelination is most active during the 3 rd trimester and throughout the 1 st yr of life $(11,12)$. It has also been demonstrated in both the rat (13-15) and human $(6,8,16)$ that the uptake of ketone bodies into brain is linearly related to the arterial concentration.

The concentrations of the ketone bodies in blood are elevated above typical adult levels both in the fetus during the later half of gestation $(8,17)$ and in the breast-fed term infant $(18)$. These data warrant considering that metabolically stable preterm infants should have a mild ketosis of similar magnitude to that of the fetus at the close of the 3 rd trimester. A recent report by Lucas et al. (18) has shown that the circulating concentrations of ketone bodies in the formula-fed term infant are significantly lower than in the breast-fed term infant. Currently, formulas developed for preterm infants contain significant amounts of MCT to increase fat digestibility (19). Since MCT are known to be ketogenic (20), premature infants may benefit from dietary MCT beyond that of enhanced fat absorption. The objective of this study was to compare plasma ketone body concentrations in infants fed three different formulas, one of which contains a significant amount of MCT.

\section{MATERIALS AND METHODS}

Twenty-three appropriate for gestational age, preterm infants with gestational ages between 30 to $35 \mathrm{wk}$ were entered into the study after informed consent was obtained. The infants were healthy and on full oral feedings, taking at least $110 \mathrm{kcal} / \mathrm{kg} / 24$ h. Two studies were performed.

Study I. Eleven infants were fed sequentially with 3 isocaloric (20 cal $/ 30 \mathrm{ml}$ ) formulas: (i) PM, (ii) SCF, and (iii) SIM. The differences in the composition of the three formulas used in this study in comparison with human milk are shown in Table 1. MCT accounts for more than $50 \%$ of the fat content of SCF. For the study, the infants were given each formula for a period of at least $96 \mathrm{~h}$. At the end of this stabilization period, samples for assay of plasma ketone bodies were obtained $2 \mathrm{~h}$ after a feeding and the plasma stored at $-20^{\circ} \mathrm{C}$ until analysis. Plasma ketone 
bodies, $3 \mathrm{HB}$ and $\mathrm{AcAc}$, were assayed according to the method of Webber with a modification of the methods described by Williamson et al. and Persson (4, 9, 21, 22).

It was later detremined that when plasma samples were stored at $-20^{\circ} \mathrm{C}$, degradation of acetoacetate occurred. Therefore, samples of plasma from rat pups were collected, pooled, and aliquoted. The plasma were frozen within $1 \mathrm{~h}$ after collection and stored at three different temperatures: at $-20^{\circ} \mathrm{C}$ in a Whirlpool Upright freezer, at $-50^{\circ} \mathrm{C}$ in a Revco Ultralow Chest Type Freezer, and at $-196^{\circ} \mathrm{C}$ in liquid nitrogen, as well as assayed for acetoacetate content without prior freezing. The plasma samples were stored frozen and after $4,7,14,21,28$ and 42 days the concentration of acetoacetate was determined. The results of these determinations are shown in Figure 1. After 30 days of storage at -20 or $-50^{\circ} \mathrm{C}$ there was greater than a $50 \%$ loss in AcAc content. Since plasma samples from infants in study I had been stored at $-20^{\circ} \mathrm{C}$ for variable periods of time prior to

Table 1. Composition of human milk and formula

\begin{tabular}{|c|c|c|c|c|}
\hline & $\begin{array}{l}\text { Human } \\
\text { milk }\end{array}$ & $\mathrm{PM}$ & SCF & SIM 20 \\
\hline \multicolumn{5}{|c|}{ A. Macronutrient composition of human milk and formula } \\
\hline \multicolumn{5}{|c|}{ Protein } \\
\hline g/liter & 11 & 15.8 & 18.3 & 15.5 \\
\hline$\%$ cal & $6 \%$ & $9 \%$ & $11 \%$ & $9 \%$ \\
\hline Source & & $\begin{array}{l}\text { Cow's milk } \\
\text { (whey } 60 \% \text { ) }\end{array}$ & $\begin{array}{l}\text { Cow's milk } \\
\text { (whey } 60 \% \text { ) }\end{array}$ & Cow's milk \\
\hline \multicolumn{5}{|l|}{ Fat } \\
\hline g/liter & 45 & 37.6 & 36.7 & 36.1 \\
\hline$\%$ cal & $55 \%$ & $50 \%$ & $47 \%$ & $48 \%$ \\
\hline Source & & $\begin{array}{l}\text { Coconut and } \\
\text { corn oil }\end{array}$ & $\begin{array}{l}\mathrm{MCT} \text {, corn and } \\
\text { coconut oil }\end{array}$ & $\begin{array}{l}\text { Coconut and } \\
\text { soy oil }\end{array}$ \\
\hline \multicolumn{5}{|c|}{ Carbohydrate } \\
\hline $\mathrm{g} /$ liter & 71 & 68.8 & 71.7 & 72.3 \\
\hline$\%$ cal & $39 \%$ & $41 \%$ & $42 \%$ & $43 \%$ \\
\hline Source & & Lactose & $\begin{array}{l}\text { Lactose and } \\
\text { corn syrup }\end{array}$ & Lactose \\
\hline \multicolumn{5}{|c|}{ B. Fatty acid composition of human milk and formula (wt $\%$ ) } \\
\hline $8: 0$ & & 3.4 & 28.1 & 2.8 \\
\hline $10: 0$ & 1.0 & 2.8 & 14.1 & 2.4 \\
\hline $12: 0$ & 4.6 & 24.2 & 10.3 & 19.4 \\
\hline 14:0 & 5.9 & 10.2 & 4.6 & 8.5 \\
\hline $16: 0$ & 21.6 & 11.0 & 6.6 & 9.6 \\
\hline $18: 0$ & 7.5 & 4.8 & 2.5 & 4.6 \\
\hline $18: 1$ & 37.0 & 16.1 & 10.7 & 14.8 \\
\hline $18: 2$ & 14.8 & 26.9 & 22.5 & 32.6 \\
\hline $18: 3$ & & 0.3 & 0.7 & 4.8 \\
\hline $18: 3+20: 1$ & 1.5 & & & \\
\hline $20: 0$ & 0.2 & 0.2 & 0.2 & 0.4 \\
\hline
\end{tabular}

determining concentrations of acetoacetate, the data are unreliable and have not been reported.

Mean body weight, gestational age, and caloric intake of the infants at birth and while they were on the different formulas are shown in Table 2A.

Study II. Study II was designed both to permit accurate determinations of acetoacetate in preterm infants under two of the three feeding conditions described above and to confirm in part the results of study I. Twelve infants were evaluated; mean body weight, gestational age, and caloric intake of the infants at birth and while they were on the different formulas are shown in Table 2B. Plasma samples were frozen immediately and stored in liquid nitrogen for no more than $6 \mathrm{wk}$ prior to analysis. Only two formulas were used sequentially, SIM and SCF. This protocol also allowed more flexibility in terms of permitting earlier discharge of the infants from the hospital.

\section{RESULTS}

Study I. Figure 2 shows the plasma concentrations of $3 \mathrm{HB}$ for infants when they were fed PM, SCF, and SIM. The plasma concentrations of $3 \mathrm{HB}$ during the PM and SIM feeding periods were similar. However the mean plasma concentrations of $3 \mathrm{HB}$ were approximately 2-fold higher when infants were fed SCF (paired Student's $t$ test, $p<0.01$ ). In addition the variability in the plasma levels of $3 \mathrm{HB}$ was greater when infants were fed SCF (range: 0.049 to $0.322 \mathrm{mM}$ ) compared to $\mathrm{PM}$ (range: 0.034 to $0.126 \mathrm{mM}$ ) and to SIM (range: 0.030 to $0.093 \mathrm{mM}$ ).

Study II. The differences in the plasma concentration of $3 \mathrm{HB}$ and ACAC in infants fed SIM and SCF formulas are shown in Figure 3. As in study I, the mean plasma concentrations of $3 \mathrm{HB}$ was significantly higher $(p<0.01)$ during SCF than during SIM feeding and again the variability in the plasma levels of $3 \mathrm{HB}$ was much greater when infants were fed SCF (range: 0.072 to 0.380 $\mathrm{mM}$ ) compared to SIM (range: 0038 to $0.099 \mathrm{mM}$ ). The values and ranges for $3 \mathrm{HB}$ were similar to those obtained in study I for the respective formulas. Concentrations of plasma AcAc in infants on SCF and SIM showed a trend similar to $3 \mathrm{HB}$. The mean

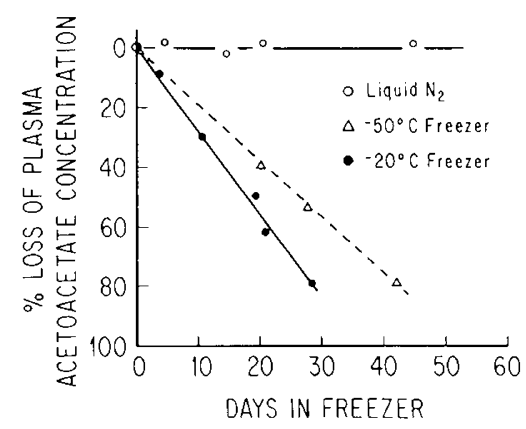

Fig. 1. Differences in the decrement of AcAc concentrations in plasma samples over time under varying storage conditions.

Table 2. Characteristics of infants in two studies (mean $\pm 1 S D$ )

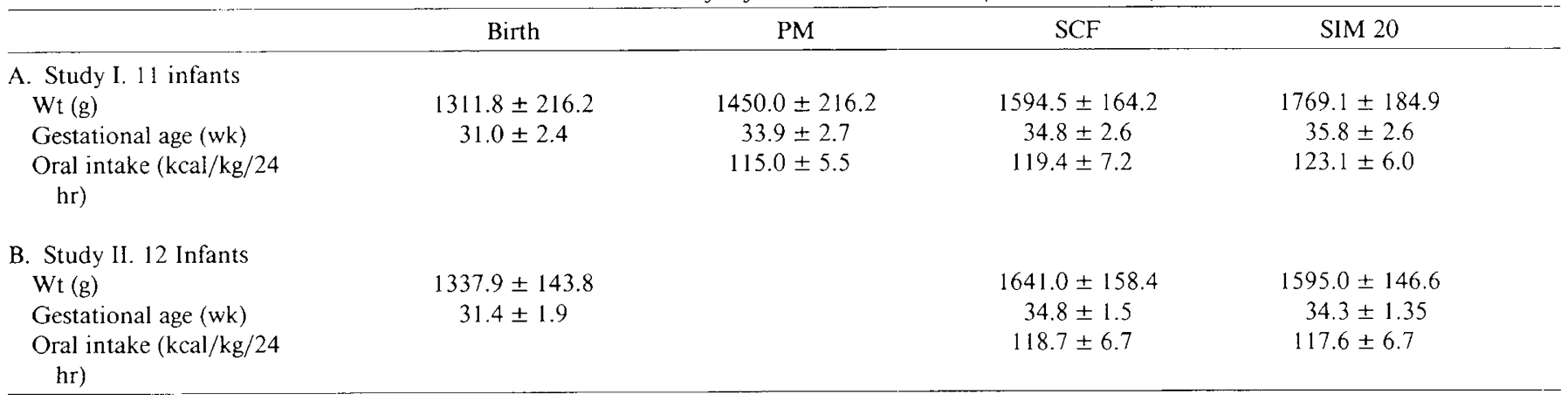




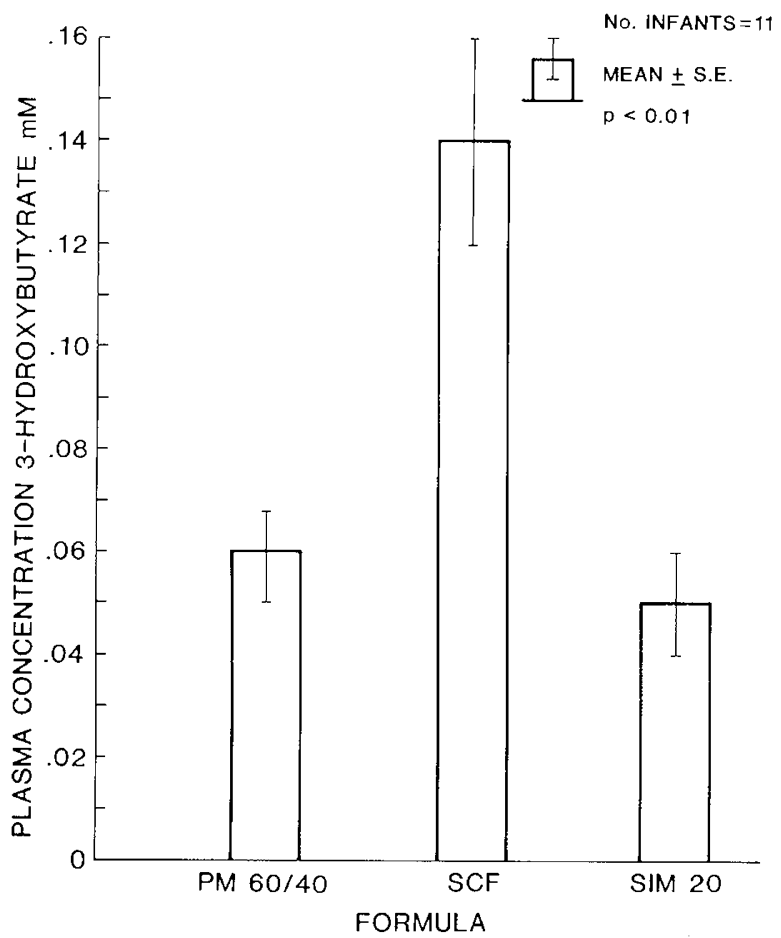

Fig. 2. Plasma concentrations of $3 \mathrm{HB}$ for infants fed PM, SCF, and SIM 20. The mean plasma concentration of $3 \mathrm{HB}$ was higher during SCF feeding than PM and SIM $(p<0.01)$.

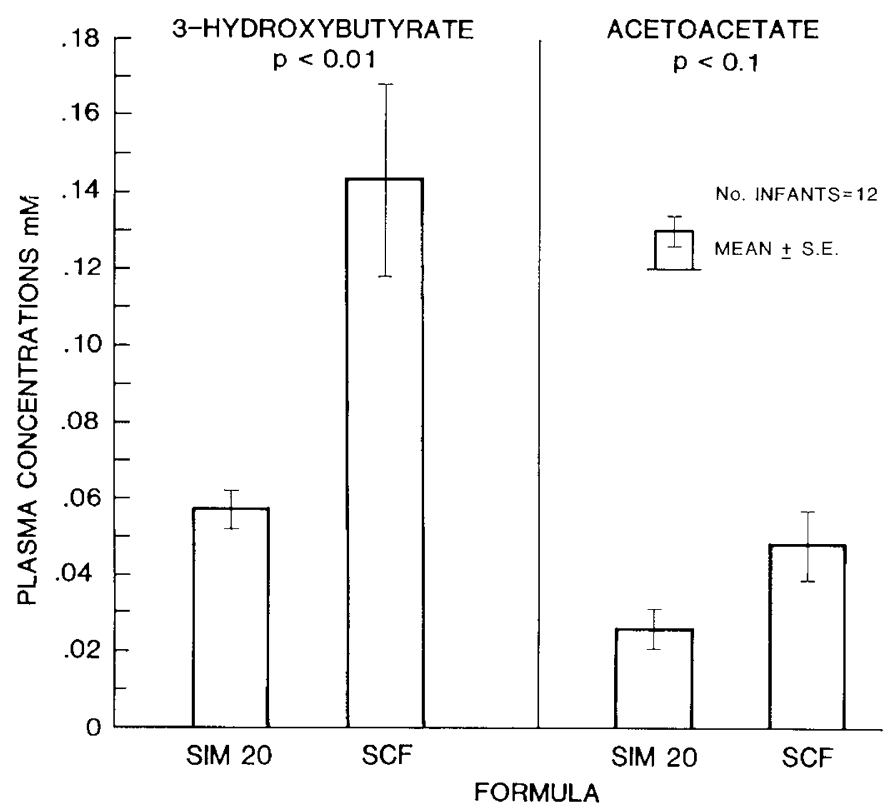

Fig. 3. Plasma concentrations of $3 \mathrm{HB}$ and $\mathrm{AcAc}$ for infants fed SIM 20 and SCF. The mean concentration of $3 \mathrm{HB}$ was significantly higher when infants were fed SCF $(p<0.01)$. Mean concentration of AcAc showed the same trend but did not reach statistical significance $(0.1>p$ $>0.05$ ).

concentration in plasma AcAc was slightly higher, although not significantly at $p<0.05$, when infants were fed SCF compared to SIM. As with $3 \mathrm{HB}$ the variability in plasma levels of AcAc was greater when infants were fed SCF (range: 0.024 to 0.110 $\mathrm{mM}$ ) compared to SIM (range: 0.007 to $0.057 \mathrm{mM}$ ).

\section{DISCUSSION}

Our study shows that feeding a milk formula containing MCT as a major component of the milk fat produces a blood ketone body concentration comparable to that of term infants who have been breast-fed (18). Whether equivalent conditions can be obtained with MCT in amounts lower than $50 \%$ of the fat in milk remains to be determined. The fatty acids of medium-chain length are octanoic, decanoic, and lauric acid. In SCF fatty acids of medium-chain length comprise $52 \%$ of the total fat, with $42 \%$ as octanoic acid and decanoic acid from MCT and $10 \%$ as lauric acid from coconut oil. On the other hand, PM and SIM contain no MCT and are rich in lauric acid from coconut oil at 24.2 and $19.4 \%$ of the total fat, respectively. It is not clear from these data whether the fatty acids in MCT are more ketogenic than lauric acid or if the optimal concentration of fatty acids of mediumchain length for promoting a mild ketosis in premature infants lies between 25 and $50 \%$ of the total fat in formula. This is an important consideration since the metabolism of the fatty acids of medium-chain length at $50 \%$ of milk fat results in the excretion of dicarboxylic acids and other hydroxyacids and their derivatives in the urine of both premature and term infants (23, 24). This may suggest that the pathways for the oxidation of medium-chain length fatty acids are overwhelmed by the substrate load, leading to their incomplete catabolism and then disposal as urinary acids. The concentrations are small since less than 2 to $3 \%$ of a dietary load of MCT at $50 \%$ of the total rat is excreted as urinary dicarboxylic acids and hydrooxyacids (25). Nevertheless, it would seem appropriate to adjust the proportion of fatty acids provided as MCT in milk fat to an amount associated with a minimal excretion of urinary dicarboxylic acid by-products while maintaining appropriate concentrations of ketone bodies in blood. Support for this recommendation can be found in reports on metabolic conditions which prevail in children with inherited defects in the oxidation of medium chain length fatty acids (26-29).

A pronounced increase in the concentration of $3 \mathrm{HB}$ in blood was observed in both studies when the formula was changed from either PM or SIM to SCF. Concentrations of acetoacetate did not change significantly. These observations are very similar to the observations made by Lucas et al. (18) who showed that the concentration of $3 \mathrm{HB}$, but not acetoacetate, was significantly higher in blood of 6-day-old term infants who were breast-fed when compared to formula-fed infants.

Since SCF contains $42 \%$ of the milk fat as MCT and because their medium-chain length fatty acids are readily metabolized to ketone bodies, we anticipated a more substantial increase in the concentrations of acetoacetate and 3HB than was found when the infants were fed SCF. To explain our observation it may be that the NADH status of the mitochondria is high, and this is reflected by the decreased ratio of [acetoacetate]:[3-hydroxybutyrate] $(18,30,31)$. In addition, the steady state concentration of acetoacetate may not increase because utilization equals the capacity for acetoacetate production. Although oxidation of lipids and synthesis of ketone bodies may be enhanced in infants fed SCF, the appropriate quantity of MCT or medium-chain length fatty acids in infant formulas necessary to promote normal growth has not been established.

As stated earlier ketone bodies, i.e. $3 \mathrm{HB}$ and AcAc, are readily available substrates for respiration and lipogenesis in the fetus. Although the mechanism of ketone body transfer across the human placenta has not been clearly defined, their concentrations in the fetus have been shown to be closely related to those in maternal plasma $(17,30-32)$. Paired umbilical venous-arterial concentrations have been measured in the fetuses of mothers whose total plasma ketone bodies were $0.2-1.4 \mathrm{mM}$ during early and advanced stages of labor (17). It was concluded from these studies that a positive maternofetal gradient exists. Thus net fetal uptake and transfetal utilization of ketone bodies appears to take place.

The mitochondria and cytosol have been found to be rich in enzymes for the metabolism of ketone bodies in both animal and human studies $(3,7,10,33,34)$. Recently, homogenous primary cultures of each of the major cell types in developing rat brain have been prepared (2). Studies on the metabolic 
capacity of these cells show that neurons, astrocytes and oligodendrocytes all have the capacity to use ketone bodies for respiration and that their capacity to use ketone bodies is much greater than their capacity to use glucose for this function. In humans, the cerebral capacity to oxidize ketone bodies is higher in neonates than in older infants. Also, the capacity of the brains of neonates and infants to remove and oxidize ketone bodies is five and four times higher than in brains of adults at a given arterial concentration $(6,13,14,16)$. The development of this capacity to utilize ketone body parallels the development of lipogenesis and myelination of the central nervous system-an essential step in the maturation process.

Another significant observation with respect to the metabolic requirements of the preterm infant comes from a recent paper by Bitman et al. (35). They report that fat in breast milk from mothers of very low birth weight infants has higher concentrations of fatty acids of medium-chain length than fat in milk from mothers of larger birth weight preterm infants, and that fat in milk from both these groups has higher concentrations of fatty acids of medium-chain length than breast milk from mothers of term infants. This difference persists until 42 days of age (35).

As we begin to appreciate the central role for ketone bodies as oxidative substrates and as precursors for lipids in the growing infant, the capacity of formulas to provide an acceptable concentration of ketone bodies in infants fed exclusively formula needs to be evaluated. At the same time, however, the potential for adverse effects due to excessive dietary amounts of MCT should also be taken into consideration. Future studies should delineate an optimal amount of MCT in formulas to achieve an appropriate ketosis with minimal production of unusual urinary dicarboxylic acids.

\section{REFERENCES}

1. Edmond $\mathbf{J} 1974$ Ketone bodies as precursors of sterols and fatty acids in the developing rat. J Biol Chem 249:72

2. Edmond J, Auestad N, Robbins RA, Bergstrom JD 1985 Ketone body metabolism in the neonate: development and the effect of diet. Fed Proc 44:23592364

3. Page MA, Krebs HA, Williamson DH 1971 Activities of enzymes of ketonebody utilization in brain and other tissues of suckling rats. Biochem J 121:4953

4. Webber RJ, Edmond J 1977 Utilization of L(+)-3-hydroxybutyrate, D(-)-3hydroxybutyrate, acetoacetate, and glucose for respiration and lipid synthesis in the 18-day-old rat. J Biol Chem 252:5222-5226

5. Adam PAJ, Raiha N, Rahiala EL, Kckomaki M 1975 Oxidation of glucose and D-beta-OH-butyrate by the early human fetal brain. Acta Paediatr Scand 64:17-24

6. Kraus H, Schlenker S, Schwedsky D 1974 Developmental changes of cerebral ketone body utilization in human infants. Hoppe-Seylers $Z$ Physiol Chem 355:164-170

7. Patel MS, Johnson CA, Rajan R, Owen OE 1975 The metabolism of ketone bodies in developing human brain: development of ketone-body utilizing enzymes and ketone bodies as precursors for lipid synthesis. $\mathbf{J}$ Neurochem 25:905-908

8. Paterson P, Sheath J, Taft P, Wood C 1967 Maternal and fetal ketone concentrations in plasma and urine. Lancet 1:862-865

9. Persson B, Gentz J 1966 The pattern of blood lipids, glycerol and ketone bodies during the neonatal period, infancy and childhood. Acta Paediatr Scand 55:353-362

10. Patel MS, Owen OE 1977 Development and regulation of lipid synthesis from ketone bodies by rat brain. J Neurochem 28:109-114
11. Himwich WA, Pennelle DK, Tucker BE 1963 Comparative biochemical development of fetal human, dog and rabbit brain. Recent Advan Biol Psychiat $5: 263$

12. Davison AN, Dobbing J 1966 Myelination as a vulnerable period in brain development. Br Med Bull 22:40-44

13. Daniel PM, Love ER, Moorehouse SR, Pratt OE, Wilson P 1971 Factors influencing utilization of ketone-bodies by brain in normal rats and rats with ketoacidosis. Lancet 11:637-638

14. Hawkins RA, Williamson DH, Krebs HA 1971 Ketone-body utilization by adult and suckling rat brain in vivo. Biochem $\mathrm{J}$ 122:13-18

15. Ruderman NB, Ross PS, Berger M. Goodman MN 1974 Regulation of glucose and ketone body metabolism in brain of anaesthetized rats. Biochem $\mathrm{J}$ $138: 1-10$

16. Persson B, Settergren G, Dahlquist G 1972 Cerebral arterio-venous difference of acetoacetate and D-beta-hydroxybutyrate in children. Acta Paediatr Scand 61:273-278

17. Sabata V Wolf $\mathrm{H}$, Lausmann S 1968 The role of free fatty acids, glycerol, ketone bodies and glucose in the energy metabolism of the mother and fetus during delivery. Biol Neonat 13:7-17

18. Lucus A, Boyes S, Bloom SR, Aynsley-Green A 1981 Metabolic and endocrine responses to a milk fed in six-day-old term infants: differences between breast and cow's milk formula feeding. Acta Paediatr Scand 70:195-200

19. Tantibhedhyangkul P, Hashim SA 1975 Medium-chain triglyceride feeding in premature infants: effects on fat and nitrogen absorption. Pediatrics 55:359369

20. Bach A, Schirardin H, Bauer M, Weryha A, 1977 Ketogenic response to medium-chain triglyceride load in the rat. J Nutr 107:1863-1870

21. Williamson DH, Lund P, Krebs HA 1967 The redox state of free nicotinamideadenine dinucleotide in the cytoplasm and mitochondria of rat liver. Biochem J 103:514-527

22. Persson B 1969 Determination of plasma acetoacetate and D-beta-hydroxybutyrate in newborn infants by an enzymatic fluorometric micro-method Scand J Clin Lab Invest 25:9-18

23. Divry P, Vianey-Liaud C, Cotte J 1984 Gas chromatography-mass spectrometry (GC-MS) diagnosis of two cases of medium chain acyl-CoA dehydrogenase deficiency. J Inherited Metabl Dis 7(suppl 1):44-47

24. Whyte RK, Hill R, McClorry S, Whelan DT 1983 Abnormal urinary fatty acids in low birthweight infants fed with medium-chain triglycerides. Proc Can Pediatr Soc 183(abstr)

25. Shigematsu Y, Momoi T, Sudo M, Suzuki Y 1982 (Omega-)-hydroxymonocarboxylic acids in urine of infants fed medium chain triglycerides. Clin Chem 27:1661-1664

26. Amedt B, Norbeck B, Moon A, Rhead W 1984 Beta-oxidation defects in man: short, medium-, and long-chain acyl-CoA dehydrogenase (ADH) deficiencies. Am J Hum Gen 36:5S

27. Duran M, Detlerk JBC, Wadman SK, Bruvinvis L, Ketting D 1984 The differential diagnosis of dicarboxylic aciduria. J Inherited Metab Dis 7(suppl 1):48-51

28. Gregersen N 1984 Fatty acyl-CoA dehydrogenase deficiency: Enzyme measurement and studies on alternative metabolism. JH Inherited Metab Dis 7 (suppl 1):28-32

29. Stanley CA, Hale DE, Coates PM, Hall CL, Corkey BE, Yang W, Kelley RI, Gonzales EL. Williamson JR, Baker L 1983 Medium-chain acyl-CoA dehydrogenase deficiency in children with nonketotic hypoglycemia and low carnitine levels. Pedaitr Res 11:877-884

30. Kim YJ, Felig P 1972 Maternal and amniotic fluid substrate levels during caloric deprivation in human pregnancy. Metabolism 21:507-512

31. Rubaltelli FF 1967 Maternal and fetal ketone levels. Lancet 1:1103

32. Sabata V, Stembera ZKS. Novak M 1968 Levels of unesterified and esterified fatty acids in umbilical blood of hypoxic fetuses. Biol Neonat 12:194-200

33. Bergstrom JD, Robbins KA, Edmond J 1982 Acetoacetyl-coenzyme A synthetase activity in rat liver cytosol. Biochem Biophys Res Commun 106:856862

34. Buckley BM, Williamson DH 1973 Acetoacetate and brain lipogenesis: Developmental pattern of acetoacetyl-coenzyme A synthetase in the soluble fraction of rat brain. Biochem $\mathrm{J}$ 132:653-656

35. Bitman J, Wood L, Hamosh M, Hamosh P. Mehta NR 1983 Comparison of lipid composition of breast milk from mothers of term and preterm infants. Am J Clin Nutr 38:300-312 